\title{
Diabetes y enfermedad vascular aterosclerótica ¿Dónde estamos?
}

\section{Diabetes and atherosclerotic vascular disease. Where do we stand?}

\author{
José Luis Díaz Díaz', Tomás Domínguez Rodríguez¹, Rosa Mª Argüeso Armesto². \\ ${ }^{1}$ Servicio de Medicina Interna. Complejo Hospitalario Universitario A Coruña. \\ ${ }^{2}$ Sección de Endocrinología. Hospital Universitario Lucus Augusti
}

La enfermedad vascular de origen aterosclerótico (EVA) comprende los episodios clínicos (agudos-críticos, recurrentes 0 crónicos) asociados a coronariopatía aterosclerótica, enfermedad cerebrovascular aterotrombótica 0 arteriopatía periférica y, constituye una de las principales causas de muerte y discapacidad en países desarrollados, especialmente entre su población con Diabetes Mellitus tipo 2 (DM2). A pesar de las múltiples campañas de promoción de hábitos de vida saludables, del creciente arsenal terapéutico para la diabetes y de la sucesión de guías de práctica clínica, la relación entre DM2 y EVA sigue siendo fatal, como demuestran los resultados del estudio DARIOS ${ }^{1}$, que analizó de forma agrupada los datos de 11 estudios de base poblacional realizados en 10 comunidades autónomas españolas durante el siglo XXI (28.887 individuos) y en cuyas conclusiones se destacaba un aumento en la prevalencia de obesidad y diabetes mellitus que se correlacionaba con la mortalidad por cardiopatía isquémica en población de 35-74 años de las diferentes comunidades autónomas, especialmente en mujeres.

Estos y otros datos justifican la presente revisión en la que recordaremos el papel de la DM2 como factor de riesgo vascular aterosclerótico (FRVA), analizaremos la magnitud de su asociación con la enfermedad vascular aterotrombótica desde una doble perspectiva, abundaremos en la controvertida categorización de su riesgo cardiovascular (alto o muy alto) y repasaremos las recomendaciones para el control de otros FRVA, para finalizar con la recopilación de datos disponibles sobre un tema candente, la seguridad cardiovascular de los antidiabéticos.

\section{Diabetes como factor de riesgo cardiovascular}

Los grandes estudios epidemiológicos de base poblacional realizados durante la segunda mitad del siglo pasado identificaron a la DM2 como FRVA. En la cohorte de Framingham, por ejemplo, la diabetes fue considerada como un factor de riesgo cardiovascular mayor e independiente incluso tras ajustar por edad avanzada, hipertensión, tabaquismo, hipercolesterolemia e hipertrofia ventricular izquierda. En dicho estudio, su presencia aumentó entre 2 y 3 veces la aparición de enfermedad vascular aterosclerótica (EVA) con un impacto relativo mayor en las mujeres². Además, el estudio MRFIT (Multiple Risk Factor Intervention Trial) reveló que, tomados de forma invidualizada, la diabetes era el FRVA de mayor peso frente a hipertensión arterial, hipercolesterolemia o tabaquismo y, que la adición de otros factores de riesgo vascular tenía un mayor efecto sobre

Gráfico 1. Riesgo relativo de enfermedad vascular aterosclerótica en diabéticos *

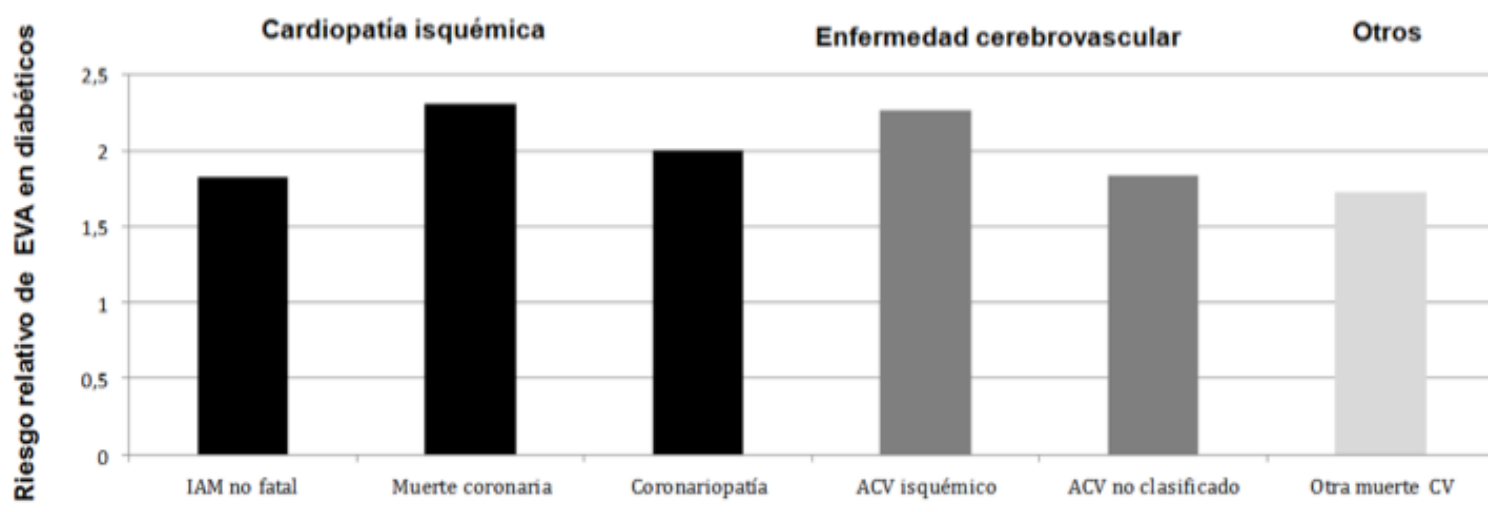

* Datos del estudio Emerging Risk Factors Collaboration ${ }^{4}$ 
la mortalidad cardiovascular entre la población diabética ${ }^{3}$. En general y teniendo en cuenta todos los territorios en donde se produce EVA, se ha calculado que la diabetes confiere el doble de riesgo, independientemente de la presencia de otros factores de riesgo convencionales ${ }^{4}$ (gráfico 1).

Diabetes y enfermedad vascular aterosclerótica: magnitud de la asociación

Según datos del reciente estudio Di@betes, la prevalencia de DM2 en población general española, se situa en el 13,8 \% ${ }^{5}$, unos 6 millones de afectados según padrón municipal de 2011; sin embargo, la prevalencia de EVA considerando todos los territorios vasculares es desconocida pues no se ha realizado estudios al respecto. Con datos de estudios epidemiológicos que analizaron la cuestión de forma parcial e incluso en ámbitos reducidos ${ }^{6-8}$ podríamos estimar que aproximadamente un $10 \%$ de la población adulta ha padecido o padece EVA.

Llegados a este punto debemos analizar cuál es la magnitud de la asociación entre diabetes y EVA y, cuestionarnos el valor de la diabetes como equivalente de EVA.

En primer lugar, la serie de estudios transversales EUROASPIRE $^{9}$ analizó entre otras variables, la prevalencia de diabetes autodeclarada entre pacientes ingresados por síndrome coronario agudo en diferentes países europeos, observando un aumento de la misma desde el $17,4 \%$ de EUROASPIRE I (1995-1996) al 28\% de EUROASPIRE III (2006-2007). Paralelamente, el Euro Heart Survey on Diabetes and the Heart, un estudio también transversal, realizado sobre más de 4.000 pacientes con síndrome coronario agudo o coronariopatía estable seleccionados en 110 centros de 25 paises europeos y, que utilizó el test de tolerancia oral a la glucosa (TTOG) para aquellos pacientes que no estaban diagnosticados previamente de diabetes detectó un $22 \%$ (pacientes agudos) y $17 \%$ (pacientes estables) de diabéticos no conocidos con una prevalencia final de DM2 del $54 \%$ y $45 \%$ respectivamente ${ }^{10}$. Con estos datos podemos concluir que aproximadamente la mitad de los pacientes con cardiopatía isquémica son diabéticos. Si eso es así, cabe preguntarse en qué proporción disminuiría la EVA si desapareciera la diabetes; en otras palabras, cuál es el riesgo poblacional de EVA atribuible a la diabetes. Desgraciadamente no tenemos datos para todos los territorios vasculares pero los estudios INTERHEART ${ }^{11} \mathrm{e}$ INTERSTROKE ${ }^{12}$ detectaron un riesgo poblacional de infarto agudo de miocardio atribuible a diabetes del $16 \%$ en mujeres y $8 \%$ en varones y, un riesgo poblacional de ictus atribuible a diabetes del $8 \%$. INTERSTROKE no analizó diferencias de riesgo por sexo, pero un estudio prospectivo realizado en el Reino Unido, sobre 244.532 sujetos de 35 a 89 años de edad (41.799 diabéticos) incluidos en la base de datos de Atención Primaria del Reino Unido (gráfico 2) y seguidos durante 7 años (1992 a 1999), evidenció un riesgo relativo de ictus aumentado entre la población diabética con un dato muy revelador, el riesgo relativo fue mayor en mujeres que en varones diabéticos (2,32 frente a 2,08), diferencia que se mantuvo para cualquier grupo de edad siendo más marcada en el grupo de 35 a 54 años de edad $(8,2 \text { frente a } 4,7)^{13}$. Por otro lado, desde la perspectiva de la propia DM2 debemos recordar que la EVA es su principal causa de muerte, sobre todo cardiopatía isquémica, en donde la coronariopatía suele ser más extensa, con menos posibilidades de revascularización y peor pronóstico, siendo frecuente las formas asintomáticas (20-40\%) especialmente cuando coexisten otros 2 factores

Gráfico 2. Riesgo relativo de ictus en diabéticos *

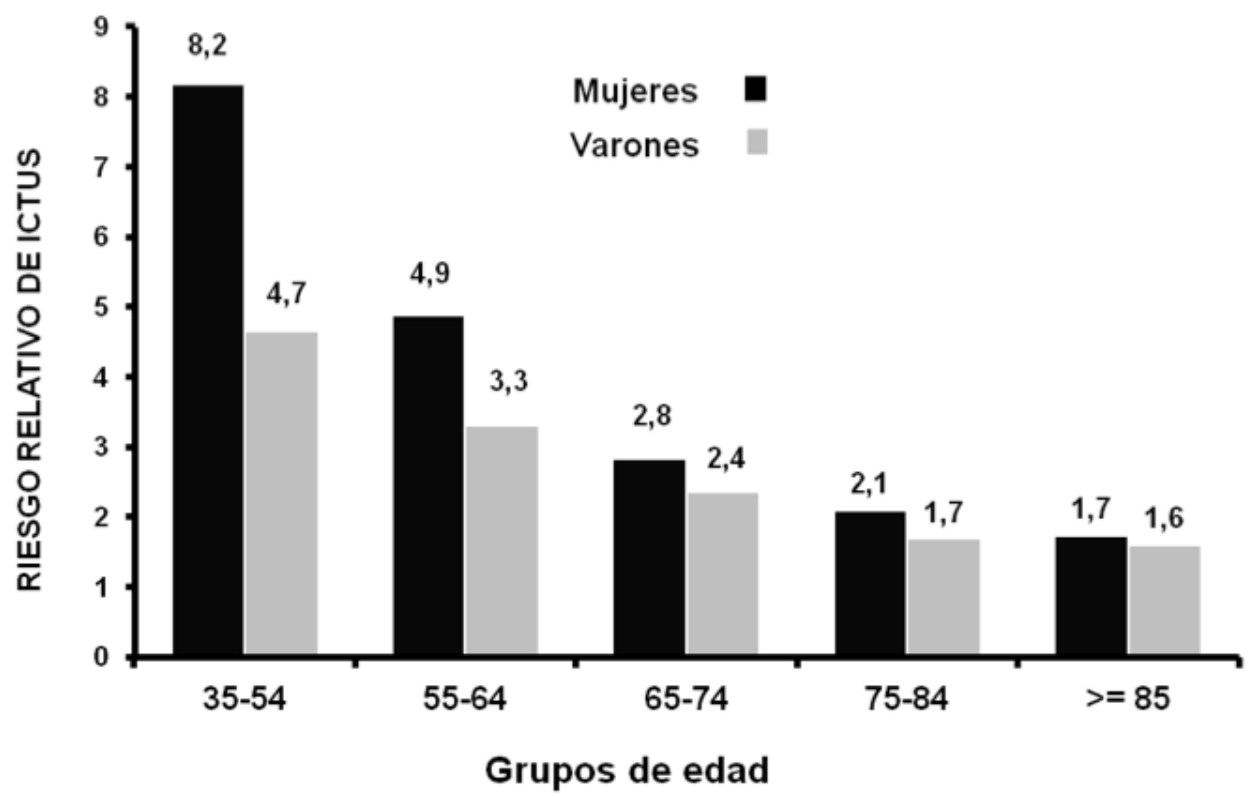

* Datos de Atención Primaria del Reino Unido ${ }^{13}$ 


\section{de riesgo cardiovascular ${ }^{14}$.}

Finalmente, repararemos en la controversia de considerar la diabetes como una situación de alto 0, como proponía Hafner, de muy alto riesgo vascular - equivalente a EVA - tras Ios resultados del ya clásico estudio finlandés de base poblacional que objetivó tras 7 años de seguimiento una incidencia de infarto agudo de miocardio (IAM) y mortalidad por coronariopatía similares entre diabéticos sin antecedentes de IAM y no diabéticos con antecedentes de $\mathrm{IAM}^{15}$, con resultados que se mantuvieron en el seguimiento a 18 años cuando el IAM fue el único criterio usado para definir EVA o incluso con mayor riesgo en población diabética, especialmente en mujeres, cuando se usaron criterios menos exigentes para definir EVA (IAM o cambios isquémicos electrocardiográficos 0 angina de pecho) ${ }^{16}$. Otros estudios, sin embargo, han dado resultados contradictorios al detectar una morbimortalidad cardiovascular menor entre los diabéticos sin antecedentes de $\mid \mathrm{AM}^{17}$, 0 una situación de equivalencia de riesgo sólo entre las mujeres diabéticas pero no en los varones ${ }^{18}$.

Un estudio realizado sobre población española tampoco ha objetivado en la diabetes una equivalencia de riesgo de EVA ${ }^{19}$. Es probable que la disparidad de resultados entre los distintos estudios pueda ser explicada por el origen y antigüedad de las distintas cohortes, el tiempo de evolución de la diabetes o los tratamientos recibidos (antidiabéticos y/o cardiovasculares) pero es seguro que la diabetes es al menos una condición de alto riesgo cardiovascular y que posiblemente adquiera la condición de muy alto riesgo cardiovascular -equivalente de EVA- en las mujeres ${ }^{20}$. Además, sabemos que la EVA aumenta proporcionalmente con el envejecimiento y que la edad de transición a alto riesgo cardiovascular se adelanta unos 15 años en la población diabética ${ }^{21}$.

\section{Lípidos, diabetes y enfermedad vascular aterosclerótica}

Aunque a menudo se hace referencia a la dislipemia diabética como un perfil lipídico genuino caracterizado por ligera elevación del colesterol plasmático a expensas del colesterol ligado a lipoproteínas de baja densidad (C-LDL), hipertrigliceridemia también ligera y descenso del colesterol ligado a lipoproteínas de alta densidad (C-HDL), lo cierto es que este fenotipo, conocido también como dislipemia aterogénica por la presencia de partículas LDL circulantes pequeñas y densas (fenotipo B), es compartido por otros trastornos como la obesidad o la Hiperlipemia Familiar Combinada en los que también subyace la insulinorresistencia. Además, en la diabetes pueden darse otros fenotipos lipídicos en función de variables que luego analizaremos.

En uno de los análisis del United Kingdom Prospective Diabetes Study (UKPDS) ${ }^{22}$ se estudió el peso de los distintos factores de riesgo para desarrollo de cardiopatía isquémica siguiendo un modelo jerárquico, y se observó que ocupaban los dos primeros lugares el C-LDL y el C-HDL, por delante de la hemoglobina $\mathrm{A}_{1} \mathrm{C}\left(\mathrm{HbA}_{1} \mathrm{C}\right)$, la presión arterial sistólica o el tabaquismo. $\mathrm{A}$ pesar de ello, es escasa y variable la información que hay sobre la prevalencia de dislipemia en población diabética, entre un 50-90\% según algunos estudios, variabilidad que se explica por lo siguiente:

- Disparidad en la definición de dislipemia: no hay ningún consenso universal que haya definido puntos de corte para identificar la normalidad de un perfil lipídico básico en población diabética.

- Evolución de la Diabetes y grado de control metabólico: en diabéticos recién diagnosticados, como eran los del UKPDS $^{23}$, se observaron solo algunas diferencias en el perfil lipídico respecto a controles sanos, consistentes en ligero descenso de C-HDL y aumento de triglicéridos y, pequeña elevación de C-LDL en mujeres diabéticas respecto a no diabéticas. La DM2 mal controlada puede cursar con hipertrigliceridemia (fenotipo IV de Friederichson) debido a un aumento en la síntesis de partículas de muy baja densidad (VLDL) y/o disminución de su catabolismo por una menor actividad del enzima lipoproteinlipasa (LPL); en sujetos genéticamente predispuestos (genotipo Apo E2/E2) también puede desencadenarse una disbetalipoproteinemia que cursa con dislipemia mixta por acúmulo de partículas de densidad intermedia (IDL).

- Tendencias demográficas y temporales que condicionan hábitos de vida y una prevalencia de obesidad variables

\section{- Pacientes ya tratados con hipolipemiantes}

La evidencia acumulada nos demuestra que los diabéticos se benefician del tratamiento hipolipemiante con estatinas de forma parecida a lo que ocurre en población no diabética: por cada Mmol/L (38 mg/dL) de descenso de C-LDL se produce una reducción de la morbimortalidad cardiovascular de un $21 \%$, beneficio que se mantiene con independencia de la presencia de otros FRVA, dislipemia aterogénica (C-HDL bajo y/o triglicéridos plasmáticos elevados) 0 la existencia de EVA previa ${ }^{24}$.

A continuación detallaremos las recomendaciones para el control lipídico en población diabética según las principales guías de práctica clínica, recomendaciones que se recogen también de forma resumida y agrupada junto a otras en la tabla 1.

- Guía de práctica clínica 2011 de la Sociedad Europea de Cardiología y Sociedad Europea de Aterosclerosis para el tratamiento de las dislipemias (ESC/EAS 2011)25: en diabéticos con enfermedad cardiovascular, nefropatía, lesión de órgano diana o mayores de 40 años con otro FRVA se recomienda como objetivo terapéutico primario un C-LDL $<70 \mathrm{mg} / \mathrm{dl}$ (clase de recomendación I, nivel de evidencia B) y, como objetivos terapéuticos secundarios, un colesterol no-HDL (diferencia entre colesterol total y C-HDL) < $100 \mathrm{mg} / \mathrm{dl}$ y un nivel de $\mathrm{ApoB}<80 \mathrm{mg} / \mathrm{dl}$ (clase de recomendación Ila, nivel de evidencia $B$ para ambos). En el resto de diabéticos se recomienda un $\mathrm{C}-\mathrm{LDL}<100 \mathrm{mg} /$ dl , colesterol no-HDL $<130 \mathrm{mg} / \mathrm{dl}$ y $\mathrm{ApoB}<100 \mathrm{mg} /$ dl. Para alcanzar tales objetivos de forma generalizada se recomienda el uso de estatinas hasta la dosis máxima recomendada o tolerada $(I, A)$. En caso de no alcanzar el objetivo terapéutico puede considerarse la asociación de 
Tabla1. Recomendaciones para objetivos de control de factores de riesgo vascular aterosclerótico en diabéticos, según guías de práctica clínica

\begin{tabular}{|c|c|c|c|c|c|c|}
\hline Objetivos & ESC/EAS 2011 & IDF 2012 & $\begin{array}{c}\text { ACC/AHA } \\
2013\end{array}$ & $\begin{array}{c}\text { ESH/ESC } \\
2013\end{array}$ & ADA 2014 & $\begin{array}{l}\text { JNC } 8 \\
2014\end{array}$ \\
\hline C-LDL (objetivo primario) & $<70 \mathrm{mg} / \mathrm{dl}^{\star}<100 \mathrm{mg} / \mathrm{dl}^{\star \star}$ & $<80 \mathrm{mg} / \mathrm{dl}$ & No objetivon & $N / A^{\star \star \star}$ & $<70 \mathrm{mg} / \mathrm{dl} \dagger<100 \mathrm{mg} / \mathrm{dl} \ddagger$ & $N / A^{\star \star \star}$ \\
\hline C no-LDL (objetivo secundario) & $<100 \mathrm{mg} / \mathrm{dl}^{\star}<130 \mathrm{mg} / \mathrm{dl}^{* *}$ & $<97 \mathrm{mg} / \mathrm{dl}$ & No objetivo & $N / A^{\star \star \star}$ & No objetivo & $N / A^{\star \star \star}$ \\
\hline ApoB (objetivo secundario) & $<100 \mathrm{mg} / \mathrm{dl}^{\star}<130 \mathrm{mg} / \mathrm{dl}^{\star \star}$ & NO objetivo & No objetivo & $N / A^{\star \star *}$ & No objetivo & $N / A^{\star \star \star}$ \\
\hline C-HDL & NO objetivo & $>39$ mg/dl & No objetivo & $N / A^{* \star \star}$ & $\begin{array}{l}>40 \mathrm{mg} / \mathrm{dl} \\
>50 \mathrm{mg} / \mathrm{dl}\end{array}$ & $N / A^{\star \star \star}$ \\
\hline Triglicéridos & NO objetivo & $<200 \mathrm{mg} / \mathrm{dl}$ & No objetivo & $N / A^{\star \star \star}$ & $<150 \mathrm{mg} / \mathrm{dl}$ & $N / A^{\star \star \star}$ \\
\hline Presión arterial $(\mathrm{mmHg})$ & $N / A^{* \star *}$ & $\leq 130 / 80$ & $N / A^{* \star *}$ & $<140 / 85$ & $<140 / 80$ & $<140 / 90$ \\
\hline $\mathrm{HbA}_{1} \mathrm{C}$ & $N / A^{\star \star \star}$ & $<7 \%$ & $N / A^{\star \star \star}$ & $N / A^{\star \star \star}$ & $<7 \%$ & $N / A^{* \star *}$ \\
\hline
\end{tabular}

* En diabéticos con enfermedad cardiovascular, nefropatía, lesión de órgano diana o mayores de 40 años con otro factor de riesgo cardiovascular. ${ }^{* \star}$ En el resto de diabéticos. ${ }^{\star \star \star}$ No aplicable IT Tratar a diabéticos de 40-75 años si C-LDL $\geq 70 \mathrm{mg} / \mathrm{dl}$ con estatinas de moderada 0 alta intensidad

estatinas con secuestradores de ácidos biliares o ezetimiba (llb, C). Si existe intolerancia a las estatinas debería considerarse el uso de secuestradores de ácidos biliares (lla, B) y/o ezetimiba (llb, C).

- Guía Global de la Diabetes 2012 de la Federación Internacional de Diabetes (IDF 2012) ${ }^{26}$ : en general se recomienda un $\mathrm{C}$-LDL $<80 \mathrm{mg} / \mathrm{dl}$, colesterol HDL > $39 \mathrm{mg} / \mathrm{dl}$, colesterol no-HDL $<97$ mg/dl y triglicéridos $<200$ mg/dl. Para alcanzar tales objetivos se recomienda el uso de estatinas aunque en caso de no alcanzar el objetivo terapéutico puede considerarse la asociación de otro hipolipemiantes como secuestradores de ácidos biliares, fibratos o ezetimiba. Si los triglicéridos son > $200 \mathrm{mg} / \mathrm{dl}$ se aconseja el tratamiento asociado con fibratos, sobre todo si hay retinopatía. No se recomienda la asociación de estatinas con gemfibrozilo. En diabéticos con EVA se recomienda como objetivo terapéutico un C-LDL $<70 \mathrm{mg} / \mathrm{dl}$.

- Guía de práctica clínica 2013 del Colegio Americano de Cardiología y la Sociedad Americana del Corazón para el tratamiento del colesterol (ACC/AHA 2013) ${ }^{27}$ : declaran insuficiente evidencia y no se pronuncian a favor ni en contra de recomendar objetivos terapéuticos de C-LDL o colesterol no-HDL (recomendación N según National Heart, Lung and Blood Institute o NHLBI); por tanto, no se definen respecto al paradigma vigente de "tratar para un nivel de C-LDL". Sin embargo, defienden "tratar desde un nivel de C-LDL" al considerar que los diabéticos tipo 2 de 40 a 75 años con C-LDL de al menos $70 \mathrm{mg} / \mathrm{dl}$ deberían ser tratados con estatinas de moderada intensidad como Atorvastatina 1020 mg, Rosuvastatina 5-10 mg, Simvastatina 20-40 mg, Pravastatina 40-80 mg, Lovastatina $40 \mathrm{mg}$, Fluvastatina XL 80 mg o Pitavastatina 2-4 mg (NHLBI grado A; ACC/ AHA grado I, A). Además, defienden que es razonable tratarlos con estatinas de alta intensidad (Atorvastatina 40-80 mg o Rosuvastatina $20 \mathrm{mg}$ ) si su riesgo cardiovascular a 10 años, calculado mediante la herramienta Pooled Co- hort Risk Assessment Equations, es de al menos un 7,5\% (NHLBI grado E; ACC/AHA grado Ila, B). Por último, contemplan que es razonable valorar el inicio, continuación 0 intensificación del tratamiento con estatinas en menos de 40 o mayores de 75 años de edad (NHLBI grado E; ACC/ AHA grado lla, C).

- Guía de la Sociedad Americana de Diabetes 2014 (ADA 2014) $)^{28}$ : abogan por la estrategia de tratar con estatinas para alcanzar un nivel de C-LDL objetivo (nivel de evidencia A), no recomendando en general su asociación con el resto de terapias hipolipemiantes por no haber demostrado un beneficio cardiovascular adicional (nivel de evidencia A). Si existe enfermedad cardiovascular evidente consideran como opción el objetivo de C-LDL $<70 \mathrm{mg} / \mathrm{dl}$, mediante el uso de estatinas a dosis elevadas (nivel de evidencia B); en su defecto recomiendan un C-LDL $<100 \mathrm{mg} / \mathrm{dL}$ (nivel de evidencia B), aunque en caso de no alcanzarse tales objetivos con la dosis máxima de estatina tolerada, una reducción del C-LDL de un 30-40\% desde niveles basales puede ser un objetivo terapéutico alternativo (nivel de evidencia B). Además, defienden que cualquier diabético con enfermedad cardiovascular manifiesta 0 mayores de 40 años con al menos otro FRVA incluído albuminuria, reciba estatinas, independientemente de su perfil lipídico (nivel de evidencia A). Por último, creen que son deseables niveles de triglicéridos < $150 \mathrm{mg} / \mathrm{dL}$ y de $\mathrm{C}-\mathrm{HDL}>50 \mathrm{mg} / \mathrm{dL}$ en mujeres y $>40 \mathrm{mg} / \mathrm{dL}$ en varones (nivel de evidencia $\mathrm{C}$ ).

Si nos centramos en los objetivos de C-LDL, en España el porcentaje de diabéticos con C-LDL $<100$ mg/dL no llega al $50 \%{ }^{29}$ y la proporción de los que alcanzan el objetivo deseado de C-LDL $<70$ mg/dL es menor del 20\%30. Más preocupante es conocer que en algún estudio, casi la mitad de los diabéticos no estaban a tratamiento con estatinas ${ }^{31}$.

\section{Presión arterial y riesgo macrovascular en diabéticos}

En la relación epidemiológica entre diabetes e hipertensión arterial (HTA) ocurre que casi la mitad de los diabéticos pa- 
decen HTA y aproximadamente la mitad de los hipertensos son diabéticos .

Estudios epidemiológicos clásicos objetivaron una relación directa y progresiva entre niveles de presión arterial (PA) Superiores a $115 / 75 \mathrm{mmHg}$ y mortalidad de origen vascular ${ }^{32}$, habiéndose calculado que por cada $10 \mathrm{mmHg}$ de descenso de PA sistólica se producía una reducción del 12\% y 19\% en el riesgo de IAM e ictus respectivamente ${ }^{33}$. El valor de la presión arterial como factor de riesgo para enfermedad vascular en población diabética es sin embargo, doble: promotor de daño "macrovascular" aterosclerótico y de enfermedad "microvascular", sobre todo nefropatía y retinopatía. En ese sentido, la presencia de HTA en población diabética eleva el riesgo relativo de ictus de 2,5 a 3,3 veces (HTA grado 1) o a 4,5 veces (HTA grado 2) respecto a población diabética no hipertensa ${ }^{34}$. De la misma forma, los diabéticos hipertensos presentan una tasa de incidencia de coronariopatía aterosclerótica sintomática que triplica la de los diabéticos normotensos ${ }^{35}$.

Esos datos han hecho recomendar durante años que para reducir la elevada morbimortalidad cardiovascular y renal, los objetivos de PA en diabéticos deberían de ser más agresivos $(<130 / 80 \mathrm{mmHg})$ que en población general no diabética $(<140 / 90 \mathrm{mmHg})$. Sin embargo, la evidencia disponible no apoya tal recomendación, pues incluso en algunos ensayos la morbimortalidad cardiovascular ha aumentado tras descensos muy acusados de la PA, haciendo reconsiderar a la comunidad científica la "curva en J", controvertido fenómeno cuya existencia o no debería de ser evaluada en un ensayo clínico exclusivo. Todo ello ha condicionado un cambio en las recomendaciones de las distintas sociedades científicas sobre el objetivo de PA en diabéticos y que exponemos a continuación (ver también tabla 1):

- Guía Global de la Diabetes 2012 de la Federación Internacional de Diabetes (IDF 2012) ${ }^{26}$ : recomiendan una PA objetivo $\leq 130 / 80 \mathrm{mmHg}$ si el tratamiento es bien tolerado. Pueden considerarse objetivos de PA menos estrictos en población anciana y cuando hay hipotensión postural 0 riesgo de caídas.

- Guía 2013 para el manejo de la hipertensión de la Sociedad Europea de Hipertensión y Sociedad Europea de Cardiología (ESH/ESC 2013) ${ }^{36}$ : basándose en los resultados de los ensayos HOT y UKPDS defienden una PA objetivo $<140 / 85$ mmHg (clase de recomendación I, nivel de evidencia A) considerándose obligado iniciar tratamiento antihipertensivo farmacológico con PA sistólica $\geq 160 \mathrm{mmHg}$ (I, A) y muy recomendado con PA sistólica $\geq 140 \mathrm{mmHg}$ (I, A). Para ello se recomienda cualquier clase de antihipertensivo aunque se prefieren los bloqueadores de sistema renina-angiotensina-aldosterona (R-A-A) especialmente en presencia de microalbuminuria o proteinuria (I, A). El doble bloqueo del sistema R-A-A, sin embargo, debería evitarse en diabéticos (III, B)

- Guía 2014 basada en la evidencia para el manejo de la presión arterial elevada en adultos (JNC 8) ${ }^{37}$ : en mayores de 18 años de edad abogan por una PA objetivo < 140/90
$\mathrm{mmHg}$ debiéndose iniciar tratamiento antihipertensivo farmacológico con $\mathrm{PA} \geq 140 / 90 \mathrm{mmHg}$ (opinión de experto grado E). Para ello recomiendan utilizar como antihipertensivo los diuréticos tiazídicos, calcioantagonistas, IECA o ARA-II (recomendación moderada grado B) pero no los betabloqueantes .

- Guía de la Sociedad Americana de Diabetes 2014 (ADA 2014) $)^{28}$ : proponen una PA objetivo $<140 / 80 \mathrm{mmHg}$ (nivel de evidencia $B$ ) aunque objetivos más estrictos (PAS $<130$ $\mathrm{mmHg}$ ) pueden plantearse en determinados pacientes, especialmente los más jóvenes, siempre que se consigan sin excesiva carga de tratamiento (nivel de evidencia C) debiéndose iniciar tratamiento antihipertensivo farmacológico con $P A \geq 140 / 80 \mathrm{mmHg}$ (B). Para ello se recomienda de elección como antihipertensivos Ios IECA o ARA-II (C) a los que se podrá asociar un calcioantagonista (amlodipino) 0 un diurético tiazídico para aquellos pacientes con un filtrado glomerular estimado (FGe)-MDRD > $30 \mathrm{ml} / \mathrm{min} / 1,73$ $\mathrm{m}^{2}, 0$ un diurético de asa en aquellos con FGe-MDRD < $30 \mathrm{ml} / \mathrm{min} / 1,73 \mathrm{~m}^{2}$ (C). Generalmente, se necesitan dos o más fármacos a dosis máximas para conseguir los objetivos (B). De ellos, al menos uno debe administrarse por la noche (A).

Siguiendo recomendaciones de la guía ESH/ESC 2013, el porcentaje de pacientes hipertensos atendidos en unidades de Atención Primaria de nuestro país con PA sistólica controlada se sitúa en el $50 \%$, el $80 \%$ tiene la PA diastólica controlada y en el $47 \%$ ambos parámetros lo están ${ }^{38}$.

\section{Tabaquismo en los diabéticos}

El consumo de tabaco es un FRVA independiente en diabéticos, existiendo al igual que en población general una correlación directa entre la intensidad del hábito y la morbimortalidad cardiovascular y general.

Como tal y según estudios de base poblacional, en diabéticos el consumo de tabaco duplica el riesgo de coronariopatía y aumenta el riesgo de ictus un $29 \%{ }^{13}$.

Todo ello tiene un especial impacto en las mujeres diabéticas tal y como evidenció el estudio Nurses' Health Study en el que se siguieron a 7.401 mujeres diabéticas durante 20 años comprobando que el riesgo de mortalidad aumentaba paralelamente al número de cigarrillos fumados por día, pasando de un riesgo relativo (RR) de 1,4 en las que nunca fumaron a un RR de 2,1 en las que fumaban más de 35 cigarrillos/ día, decreciendo sustancialmente hasta un RR de 1,1 tras 10 años de abandono del hábito tabáquico ${ }^{39}$. Del mismo modo, en diabéticas que fumaban $\geq 15$ cigarrillos/día, el RR de coronariopatía aterosclerótica aumentaba a 2.68 respecto a las que nunca habían fumado, volviendo a equipararse los riesgos tras 10 años de abandono del tabaquismo (RR 1,01$)^{40}$.

Dado que la población diabética tiene un riesgo intrínseco aumentado de EVA, resulta de especial interés el abandono del tabaco como medida esencial en la prevención y tratamiento de dicha población y así lo reflejan las distintas guías de práctica clínica ${ }^{26,28}$. 


\section{Control glucémico y riesgo vascular aterosclerótico}

La Diabetes Mellitus se ha definido como un trastorno o conjunto de trastornos que tienen como rasgo común y principal la hiperglucemia, considerada como tal un punto de corte a partir del cual aumenta el riesgo de microangiopatía, sobre todo retinopatía. Desde ese aspecto conceptual la diabetes había sido inicialmente entendida como un proceso de riesgo microvascular, pero tanto la hiperglucemia per se como el elenco de otros FRVA que a menudo aglutina, le confieren un marcado carácter de riesgo macrovascular. En efecto, el aumento de la glucemia plasmática, tanto si se mide en situación de ayuno como tras un test de sobrecarga oral de glucosa, se ha correlacionado directamente con la incidencia de accidentes cardiovasculares ${ }^{41}$. En esa misma dirección, se sabe que por cada $1 \%$ de aumento de la $\mathrm{HbA}_{1} \mathrm{c}$ se produce un aumento del $18 \%$ del RR de enfermedad cardiovascular ${ }^{42}$.

Llegados a este punto la cuestión es conocer si mejorando el control glucémico podemos disminuir el riesgo de EVA y hasta qué punto, control menos o más intensivo, se mantiene tal beneficio. Paralelamente habría que considerar si ese potencial beneficio depende más del antidiabético utilizado que del grado de control glucémico alcanzado, cuestión que exploraremos en el siguiente apartado de seguridad cardiovascular de los antidiabéticos.

El ensayo UKPDS43 iniciado en 1.977 testó la hipótesis de que el control glucémico intensivo respecto al control estándar de entonces reportaba beneficios en términos de reducción de la morbimortalidad micro y macrovascular. Para ello, se incluyeron 4.209 diabéticos recién diagnosticados (glucemia en ayunas repetida $>6 \mathrm{mmol} / \mathrm{l} 0108 \mathrm{mg} / \mathrm{dl}$ ) que fueron randomizados a cualquiera de las 2 estrategias señaladas demostrando que, tras 10 años de seguimiento, los pacientes del brazo de tratamiento intensivo (mediana $\mathrm{HbA}_{1} \mathrm{C} 7 \%$ ) respecto a los del tratamiento estándar (mediana $\mathrm{HbA}_{1} \mathrm{C} 7,9 \%$ ) presentaban una reducción estadísticamente significativa del $12 \%$ en el RR de cualquier complicación relacionada con la diabetes, beneficio que se debía en gran medida al descenso del $25 \%$ en el RR de complicaciones microvasculares, pero no de las macrovasculares. En esta extrapolación de resultados se basa la consideración de $\mathrm{HbA}_{1} \mathrm{C} 7 \%$ como un objetivo adecuado de control metabólico en diabéticos.

Sin embargo, la población diabética sigue teniendo complicaciones por lo que en la pasada década se pusieron en marcha otros ensayos clínicos como el VADT, ACCORD, o ADVANCE, en diabéticos con mayor tiempo de evolución, para valorar el impacto del control metabólico más intensivo. Con niveles finales de $\mathrm{HbA}_{1} \mathrm{C}$ de $6,9 \%, 6,4 \%$ y 6,5\% respectivamente, hubo beneficio parcial y significativo en la reducción de algún componente microvascular (fundamentalmente microalbuminuria). Sin embargo, la reducción del RR de EVA fue de escasa magnitud $(12 \%, 10 \%$ y $6 \%$ respectivamente) y no significativa; más aún, en el ensayo ACCORD y en un análisis de subgrupos del VADT se observó, en la rama de tratamiento intensivo, un aumento de la mortalidad todavía no aclarado. Un metaanálisis ${ }^{44}$ que agrupó esos y otros ensayos concluyó que el control glucémico intensivo no modifica la mortalidad general ni la morbimortalidad cardiovascular y que, aunque consigue una reducción del $12 \%$ en el RR de enfermedad microvascular, también aumenta un $30 \%$ el RR de hipoglucemia por lo que el balance riesgo/beneficio puede ser en algunos pacientes desfavorable.

Así pues, los objetivos de control glucémico según guías de práctica clínica y que detallamos a continuación, tienen en cuenta el beneficio esperado en la reducción de la morbimortalidad micro y macrovascular de forma conjunta.

- Guía Global de la Diabetes 2012 de la Federación Internacional de Diabetes (IDF 2012) ${ }^{26}$ : aconseja el mantenimiento de una $\mathrm{HbA}_{1} \mathrm{C}<7,0 \%$ puesto que minimiza el riesgo de desarrollar complicaciones. Un objetivo de HbA1c inferior puede ser considerado si se alcanza con facilidad y seguridad. Según la guía, un objetivo de $\mathrm{HbA}_{1} \mathrm{C}$ mayor puede ser considerado diabéticos con comorbilidades o cuando los intentos anteriores para optimizar el control se han asociado con hipoglucemias inaceptables.

- Guía de la Sociedad Americana de Diabetes 2014 (ADA 2014) ${ }^{28}$ : consideran razonable en adultos no embarazados un objetivo de $\mathrm{HbA}_{1} \mathrm{C}<7 \%$ (nivel de evidencia B) aunque se podría intentar conseguir objetivos más ambiciosos $\left(\mathrm{HbA}_{1} \mathrm{C}<6,5 \%\right)$ siempre que se alcancen sin hipoglucemias, en diabéticos seleccionados como aquellos con corta duración de la diabetes, larga expectativa de vida y sin enfermedad cardiovascular manifiesta (nivel de evidencia C). Para pacientes con historia de hipoglucemias, larga duración de la diabetes, corta expectativa de vida, comorbilidad, enfermedad micro y/o macrovascular y que reciben politerapia a dosis eficaz, pueden ser más apropiados objetivos menos rigurosos, recomendándose una $\mathrm{HbA}_{1} \mathrm{C}<8 \%$ (nivel de evidencia $\mathrm{B}$ )

A pesar de quedar demostrado que un objetivo general de $\mathrm{HbA}_{1} \mathrm{C} 7 \%$ es adecuado para población diabética, un estudio realizado en nuestra comunidad, el proyecto OBTEDIGA ${ }^{45}$, nos alertó de que sólo un $54,3 \%$ de los diabéticos atendidos en el ámbito de la Atención Primaria, alcanzaban tal objetivo.

\section{Seguridad cardiovascular de los antidiabéticos}

La historia de la terapéutica antidiabética en relación con sus beneficios "macrovasculares" ha estado salpicada de luces y sombras.

El subgrupo de diabéticos con sobrepeso del UKPDS que fueron asignados al brazo de tratamiento intensivo y recibieron metformina, experimentaron una reducción del 32\% en el RR de cualquier complicación relacionada con la diabetes, reducción del $36 \%$ de la mortalidad por cualquier causa y del $39 \%$ de infarto agudo de miocardio ${ }^{46}$, beneficios que se mantuvieron a modo de "legado metabólico" incluso 10 años después de haber finalizado el estudio y una vez que los pacientes estaban siendo tratados en condiciones de práctica clínica habitual ${ }^{47}$. Posteriormente, en el ensayo PROactive realizado en diabéticos con EVA, pioglitazona demostró frente a placebo - y otros antidiabéticos- una reducción del 16\% 
Tabla2. Recomendaciones de la Food and Drug Administration sobre requisitos de seguridad cardiovascular de los nuevos antidiabéticos 2008.

\begin{tabular}{|c|c|c|}
\hline \multicolumn{2}{|c|}{$\begin{array}{c}\text { Riesgo relativo de enfermedad } \\
\text { cardiovascular en metaanálisis de } \\
\text { ensayos en fase } 2 \text { y } 3\end{array}$} & CONCLUSIÓN \\
\hline & $<1.3$ & El fármaco no se aprueba \\
\cline { 2 - 3 } & $1.3-1.8$ & $\begin{array}{c}\text { Se precisa estudio aclaratorio } \\
\text { postcomercialización }\end{array}$ \\
\cline { 2 - 3 } $\begin{array}{c}\text { Límite superior } \\
\text { RR (IC 95\%) }\end{array}$ & $>1.8$ & $\begin{array}{c}\text { No precisa estudios } \\
\text { adicionales }\end{array}$ \\
\cline { 2 - 3 } & \\
\hline
\end{tabular}

en el objetivo secundario compuesto de muerte por cualquier causa, IAM no fatal e ictus, pero no en el primario, objetivo que tampoco se alcanzó en otro ensayo con pioglitazona realizado en Japón (PROFIT-J).

En ese contexto entramos en "el lío de la rosiglitazona" tras la publicación del controvertido metaanálisis de Nissen en 2007 que relacionaba dicho fármaco con un aumento del $43 \%$ en el RR de IAM y una tendencia no significativa al aumento de la mortalidad cardiovascular. Eso significó un antes y un después en la historia de los antidiabéticos pues en el año 2008 la Food and Drug Administration (FDA) emitió un informe en el que se recogían una serie de consideraciones para la aprobación de nuevos antidiabéticos. En ese sentido, la FDA entendía que el requisito para aprobación de un antidiabético era la eficacia en la reducción de $\mathrm{HbA}_{1} \mathrm{C}$ y no precisaba demostrar reducción del riesgo de enfermedad cardiovascular, pero su análisis debía de incluirse en ensayos clínicos de fase 2-3 y demostrar que el nuevo fármaco no lo aumentaba (ver tabla 2). Por tanto, se dejaba de hablar de "beneficio cardiovascular" y se pasaba a hablar de "seguridad cardiovascular". Rosiglitazona fue retirada de Europa y la FDA impuso importantes restricciones de uso hasta que fueron eliminadas a finales del año 2013, tras los resultados del ensayo RECORD ${ }^{49}$ en el que rosiglitazona, frente a sulfonilureas (ambas en asociación con metformina), no aumentaba la morbimortalidad cardiovascular.

Existe inquietud sobre fármacos ya comercializados como las sulfonilureas y había muchas dudas sobre la seguridad cardiovascular del uso de insulinas, dudas que quedaron disipadas en parte tras la publicación de los resultados del ensayo Origin en el que insulina glargina, administrada a una dosis media de 0,3-0,4 U/kg/día para un objetivo de glucemia basal $<95 \mathrm{mg} / \mathrm{dL}$, demostró un efecto neutro sobre la morbimortalidad cardiovascular frente al tratamiento estándar y tras una mediana de seguimiento de 6,2 años.

De los fármacos comercializados en los últimos años tenemos ya resultados de seguridad cardiovascular de algunos inhibidores de la dipeptidilpeptidasa-4 (IDPP-4) como alogliptina (estudio EXAMINE) y saxagliptina (estudio SAVOR-TIMI 53), mostrando ambos un efecto neutro, si bien en el caso de saxagliptina hubo un aumento todavía no aclarado del 27\% en la hospitalización por insuficiencia cardíaca.
Quedamos a la espera de conocer la seguridad cardiovascular testada en los estudios en marcha para otros IDDP-4 como sitagliptina (TECOS) o linagliptina (CAROLINA) pero no vildagliptina. Igualmente están siendo testados los análogos del GLP-1 como liraglutida (LEADER), exenatida LAR (EXSCEL) o lixisenatida (ELIXA) y, los inhibidores del cotransportador de glucosa tipo 2 dependiente del sodio (SGLT-2) como dapagliflozina (DECLARE-TIMI-58), canagliflozina (CANVAS) y empagliflozina (NCT01131676). Todos ellos ayudarán, en cierta medida, a individualizar la terapia antidiabética y aclarar también si en los limitados beneficios del tratamiento glucémico intensivo influye el antidiabético elegido.

\section{Conclusiones}

La diabetes mellitus es una condición que duplica el riesgo de enfermedad vascular aterosclerótica para cualquier territorio, llegando en algunos casos como en las mujeres a constituir un verdadero "equivalente de riesgo". En esa relación, un 50\% de quienes padecen coronariopatía manifiesta son diabéticos y, la mayoría de los diabéticos fallecerán por complicaciones macrovasculares. Es por ello esencial el control de los distintos factores de riesgo vascular aterosclerótico que a menudo coexiste en la población diabética - además de la hiperglucemia-, control que apenas se consigue en la mitad de los diabéticos. El panorama no es alentador, a no ser que interioricemos en nuestra práctica clínica el control integral del riesgo macro y microvascular cuyos tremendos beneficios quedaron demostrados con los resultados del estudio STENO y de su "legado metabólico" 50 .

\section{Bibliografía}

1. Grau M, Elosua R, Cabrera de León A, Guembe MJ, Baena-Diez JM, Vega Alonso T, Felix FJ, Zorrilla B, Rigo F, Lapetra J, Gavrila D, Segura A, Sanz H, Fernández-Bergés $D$, Fito $M$, Marrugat J. Factores de riesgo cardiovascular en España en la primera década del siglo XXI: análisis agrupado con datos individuales de 11 estudios de base poblacional. Estudio DARIOS. Rev Esp Cardiol 2011; 64(4): 295-304

2. Kannel WB, MCGee DL. Diabetes and Cardiovascular Disease.The Framingham Study JAMA. $1979 ; 241(19): 2035-2038$

3. Stamler J, Vaccaro 0, Neaton JD, Wentworth D. Diabetes, other risk factors, and 12 yr cardiovascular mortality for men screened in the Multiple Risk Factor Intervention Trial. Diabetes Care 1993;16:434-444.

4. Emerging Risk Factors Collaboration, Sarwar N, Gao P, et al. Diabetes mellitus, fasting blood glucose concentration, and risk of vascular disease: a collaborative metaanalysis of 102 prospective studies. Lancet 2010; 375:2215.

5. Soriguer F, Soriguer F, Goday A, et al. Prevalence of diabetes mellitus and impaired glucose regulation in Spain: the Di@bet.es Study. Diabetologia. 2012 Jan;55(1):8893.

6. Bermejo, F.; Vega, S.; Morales, J. et al. Prevalence of stroke in two samples (rural and urban) of old people in Spain. A pilot door-to-door study carried out by health professionals. Neurología 1997; 4:157-161.

7. López-Bescos L, Cosín J, Elosúa R, Cabadés A, de los Reyes M, Arós F et al. Prevalencia de angina y factores de riesgo cardiovascular en las diferentes comunidades autónomas de España: estudio PANES. Rev Esp Cardiol 1999;52: 1045-1056.

8. Ramos R, Quesada M, Solanas P, Subirana I, Sala J, Vila J, et al, on behalf of the REGICOR Investigators. Prevalence of symptomatic and asymptomatic peripheral arterial disease and the value of the ankle-brachial index to stratify cardiovascular risk. Eur J Vasc Endovasc Surg. 2009; 38:305-311

9. Kotseva K, Wood D, De Backeret G, et al, for the EUROASPIRE Study Group. Cardiovascular prevention guidelines in daily practice: a comparison of EUROASPIRE I, II and III surveys in eight European countries. Lancet 2009; 373: 929-940

10. Bartnik M, Rydén L, Ferrari R, Malmberg K, Pyörälä K, Simoons ML, Standl E, SolerSoler J, Öhrvik J, on behalf of the Euro Heart Survey Investigators. The prevalence of abnormal glucose regulation in patients with coronary artery disease across Europe. Eur Heart J 2004;25:1880-1890.

11. Yusuf S, Hawken S, Ounpuu S, et al, for the INTERHEART Study Investigators. Effect 
of potentially modifiable risk factors associated with myocardial infarction in 52 countries (the INTERHEART study): a case-control study. Lancet. 2004;364:937-952.

12. O'Donnell MJ, Xavier D, Liu L, Zhang H, Chin SL, Rao-Melacini P, Rangarajan S, Islam S, Pais P, McQueen MJ, Mondo C, Damasceno A, Lopez-Jaramillo P, Hankey GJ, Dans AL, Yusoff K, Truelsen T, Diener HC, Sacco RL, Ryglewicz D, Czlonkowska A, Weimar C, Wang X, Yusuf S, \& INTERSTROKE investigators (2010). Risk factors for ischaemic and intracerebral haemorrhagic stroke in 22 countries (the INTERSTROKE study): a case-control study. Lancet, 2010;376 (9735):112-123

13. Mulnier H; Seaman H; Raleigh V; Soedamah-Muthu S; Colhoun Lawrenson; Vries C. Risk of stroke in people with type 2 diabetes in the UK: a study using the General Practice Research Database. Diabetologia, 2006; 49(12):2859-2865

14. Scognamiglio R, Negut $C$, Ramondo $A$, et al. Detection of coronary artery disease in asymptomatic patients with type 2 diabetes mellitus. J Am Coll Cardiol 2006; 47:65

15. Haffner S M, Lehto S, Rönenemaa T et al. Mortality from coronary heart disease in subjects with type 2 diabetes and in non diabetic subjects with and without prior myocardial infarction. N Engl J Med 1998; 339:229 -234

16. Juutilainen $A$, Lehto $S$, Rönnemaa T, Pyörälä $K$, Laakso $M$. Type 2 diabetes as a "coronary heart disease equivalent": an 18-year prospective population-based study in Finnish subjects. Diabetes Care. 2005 Dec;28(12):2901-2907.

17. Vaccaro 0, Eberly LE, Neaton JD, Yang L, Riccardi G, Stamler J: Impact of diabetes and previous myocardial infarction on long-term survival: 25-year mortality follow-up of primary screenees of the Multiple Risk Factor Intervention Trial. Arch Intern Med 2004;164:1438-1443

18. Becker A, Bos G, de Vegt F, Kostense PJ, Dekker JM, Nijpels G, Heine RJ, Bouter LM, Stehouwer, CDA: Cardiovascular events in type 2 diabetes: comparison with nondiabetic individuals without and with prior cardiovascular disease: 10-year follow-up of the Hoorn Study. Eur Heart J 2003;24:1406-1413

19. Cano JF, Baena-Diez JM, Franch J, Vila J, Tello S, Sala J, Elosua R and Marrugat $\mathrm{J}$, on behalf of the REGICOR and GEDAPS Investigators. Long-Term Cardiovascular Risk in Type 2 Diabetic Compared With Nondiabetic First Acute Myocardial Infarction Patients: A population-based cohort study in southern Europe. Diabetes Care 2010; 33:2004-2009

20. Lee C, Joseph L, Colosimo A, Dasgupta K. Mortality in diabetes compared with previous cardiovascular disease: a gender-specific meta-analysis. Diabetes Metab. 2012 Nov;38(5):420-427.

21. Booth GL, Kapral MK, Fung K, Tu JV. Relation between age and cardiovascular disease in men and women with diabetes compared with non-diabetic people: a population-based retrospective cohort study. Lancet 2006; 368:29.

22. Turner RC, Millns H, Neil HAW, et al. Risk factors for coronary artery disease in noninsulin dependent diabetes mellitus: United Kingdom Prospective Diabetes Study (UKPDS: 23). BMJ. 1998;316:823-828.

23. U.K. Prospective Diabetes Study Group. U.K. Prospective Diabetes Study 27: plasma lipids and lipoproteins at diagnosis of NIDDM by age and sex. Diabetes Care 1997;20:1683-1687

24. Kearney PM, Blackwell L, Collins R, Keech A, Simes J, Baigent C, Cholestero Treatment Trialists' (CTT) Collaborators: Efficacy of cholesterol-lowering therapy in 18,686 people with diabetes in 14 randomised trials of statins: a meta-analysis. Lancet 2008; 371(9607):117-125

25. Task Force for the management of dyslipidaemias of the European Society of Cardiology (ESC) and the European Atherosclerosis Society (EAS), Catapano AL, Reiner Z, De Backer G, Graham I, Taskinen MR, et al.; ESC Committee for Practice. ESC/ EAS Guidelines for the management of dyslipidaemias: the Task Force for the management of dyslipidaemias of the European Society of Cardiology (ESC) and the European Atherosclerosis Society (EAS). Atherosclerosis 2011;217 Suppl 1:S1-44.

26. IDF Clinical Guidelines Task Force. Global Guideline for Type 2 Diabetes. Brussels: International Diabetes Federation, 2012

27. Stone NJ, Robinson J, Lichtenstein AH, Merz CN, Blum CB, Eckel RH, et al. 2013 ACC/AHA Guideline on the Treatment of Blood Cholesterol to Reduce Atherosclerotic Cardiovascular Risk in Adults: A Report of the American College of Cardiology/ American Heart Association Task Force on Practice Guidelines. Circulation 2014 Jun 24;129(25 Suppl 2):S1-S45

28. Standards of Medical Care in Diabetes 2014. Diabetes Care 2014;37:S14-80.

29. Rodríguez-Roca GC, Llisterri JL, Barrios V, Alonso-Moreno FJ, Banegas JR, Lou S, Matalí A. Grado de control de la dislipemia en los pacientes españoles asistidos en atención primaria. Estudio LIPICAP. Clin Invest Arterioscl. 2006;18:226-238.
30. Pérez de Isla L, Saltijeral Cerezo A, Vitale G, González Timón B, Torres Do Rego A, Alvarez-Sala Walther LA. Prevalencia de colesterol LDL inadecuado en pacientes con enfermedad coronaria y/o diabetes mellitus tipo 2. Rev Clin Esp 2012;212(10):475481

31. Robles R, Barroso S, Marcos G, Sánchez F. Control de la dislipemia en pacientes diabéticos en Extremadura. Endocrinología y Nutrición, 2009; 56(3):112-117

32. Lewington S, Clarke R, Qizilbash N, Peto R, Collins R. Age-specific relevance of usua blood pressure to vascular mortality: a metaanalysis of individual data for one million adults in 61 prospective studies. Lancet 2002; 360:1903-1913.

33. Adler Al, Stratton IM, Neil HA, et al. Association of systolic blood pressure with macrovascular and microvascular complications of type 2 diabetes (UKPDS 36): prospective observational study. BMJ. 2000;321(7258):412-419.

34. Hu G, Sarti C, Jousilahti P, Peltonen M, Qiao Q, Antikainen R, Tuomilehto J. The impact of history of hypertension and type 2 diabetes at baseline on the incidence of stroke and stroke mortality. Stroke. 2005 Dec;36(12):2538-2543.

35. Assman G, Cullen P, Schulte H. Simple scoring scheme for calculating the risk of acute coronary events based on the 10-year follow-up of the prospective cardiovascular Münster (PROCAM) study. Circulation. 2002;105:310-315.

36. Mancia G, Fagard R, Narkiewicz K, et al. 2013 ESH/ESC guidelines for the management of arterial hypertension: the Task Force for the Management of Arterial Hypertension of the European Society of Hypertension (ESH) and of the European Society of Cardiology (ESC). Eur Heart J. 2013;34(28):2159-2219

37. James PA, Oparil S, Carter BL, et al. Evidence based-guideline for the management of high blood pressure in adults: Report from the panel members appointed to the Eighth Joint National Committee (JNC 8). JAMA 2014; 311(5):507-520

38. Llisterri JL, Rodríguez G, Pérez MA, et al., en representación del Grupo de Trabajo de Hipertensión Arterial de la Sociedad Española de Médicos de Atención Primaria (Grupo HTA/SEMERGEN) y de los investigadores del Estudio PRESCAP 2006. Contro de la presión arterial en una población hipertensa diabética asistida en atención primaria. Estudio PRESCAP-Diabetes. Rev Semergen. 2010; 36:307-316.

39. Al-Delaimy WK, Willett WC, Manson JE, Speizer FE, Hu FB. Smoking and mortality among women with type 2 diabetes: the Nurses' Health Study cohort. Diabetes Care. 2001;24(12):2043-2048

40. Al-Delaimy WK, Manson JE, Solomon CG, Kawachi I, Stampfer MJ, Willett WC, Hu FB. Smoking and risk of coronary heart disease among women with type 2 diabetes mellitus. Archives of Internal Medicine. 2002;162(3):273-279.

41. Coutinho $\mathrm{M}$ et al. The relationship between glucose and incident cardiovascular events. A metaregression analysis of published data from 20 studies of 95,783 individuals followed for 12.4 years. Diabetes Care 1999;22:233-240.

42. Selvin E, Marinopoulos S, Berkenblit G, et al. Meta-analysis: glycosylated hemoglobin and cardiovascular disease in diabetes mellitus. Ann Intern Med 2004; 141:421.

43. UK Prospective Diabetes Study (UKPDS) Group. Intensive blood-glucose control with sulphonylureas or insulin compared with conventional treatment and risk of complications in patients with type 2 diabetes (UKPDS 33). Lancet. 1998;352(9131):837853

44. Hemmingsen B, Lund SS, Gluud C, et al. Intensive glycaemic control for patients with type 2 diabetes: systematic review with meta-analysis and trial sequential analysis of randomised clinical trials. BMJ. 2011 Nov 24;343:d6898.

45. Vidal Pardo JI, Pérez Castro TR, López Álvarez XL, García Soidán FJ, Santiago Pérez MI, Muñiz J. Quality of care of patients with type-2 diabetes in Galicia (NW Spain) [OBTEDIGA project]. Int J Clin Pract. 2011; 10: 1067-1075

46. Effect of intensive blood-glucose control with metformin on complications in overweight patients with type 2 diabetes (UKPDS 34). UK Prospective Diabetes Study (UKPDS) Group. Lancet 1998 Sep 12; 352(9131):854-865

47. Holman RR, Paul SK, Bethel MA, et al. 10-year follow-up of intensive glucose contro in type 2 diabetes. N Engl J Med 2008; 359:1577

48. Nissen SE, Wolski K. Effect of rosiglitazone on the risk of myocardial infarction and death from cardiovascular causes. N Engl J Med 2007;356:2457-2471

49. Home PD, Pocock SJ, Beck-Nielsen H, et al. Rosiglitazone evaluated for cardiovascular outcomes in oral agent combination therapy for type 2 diabetes (RECORD): a multicentre, randomised, open-label trial. Lancet 2009;373:2125-2135

50. Gaede $P$, Lund-Andersen $H$, Parving $H H$, Pedersen 0 . Effect of a multifactorial intervention on mortality in type 2 diabetes. N Engl J Med. 2008 ;358(6):580-591 